\title{
Estresse no trabalho em enfermeiros brasileiros atuantes na atenção primária à
}

\section{saúde}

\author{
Stress at work in brazilian nurses acting in primary health care \\ Estrés en el trabajo en enfermeras brasileñas que actúan en atención primaria de salud
}

Recebido: 01/03/2021 | Revisado: 10/03/2021 | Aceito: 01/06/2021 | Publicado: 17/06/2021

\author{
Ewellyn Cristine dos Santos \\ ORCID: https://orcid.org/0000-0001-9144-8035 \\ Instituto Virmond de Guarapuava, Brasil \\ E-mail: ewellyn.cristine71@gmail.com \\ Kátia Pereira de Borba \\ ORCID: https://orcid.org/0000-0003-2164-4289 \\ Universidade Estadual do Centro Oeste, Brasil \\ E-mail: kborba@unicentro.br \\ Marília Daniella Machado Araújo Cavalcante \\ ORCID: https://orcid.org/0000-0002-7685-6679 \\ Universidade Estadual do Centro Oeste, Brasil \\ E-mail: maraujo@unicentro.br \\ Daniela Viganó Zanoti-Jeronymo \\ ORCID:https://orcid.org/0000-0002-6131-3890 \\ Universidade Estadual do Centro Oeste, Brasil \\ E-mail: danielazanoti@uol.com.br \\ Vania Gryczak \\ ORCID: https://orcid.org/0000-0001-9831-0282 \\ Universidade Estadual do Centro Oeste, Brasil \\ E-mail: vaniag@unicentro.br \\ Rodolfo Pereira de Borba \\ ORCID: https://orcid.org/0000-0002-4169-7997 \\ Centro Universitário Campo Real, Brasil \\ E-mail:rodolfo.borba@gmail.com
}

\begin{abstract}
Resumo
Introdução: Enfermeiros atuantes na atenção primária à saúde são profissionais potencialmente susceptíveis ao desenvolvimento de estresse. Em seu cotidiano, enfrentam situações conflituosas como excesso de trabalho e acúmulo de tarefas que causam desgaste físico e mental. Objetivo: Analisar a percepção de enfermeiros brasileiros atuantes na atenção primária à saúde sobre a contribuição do estresse no ambiente de trabalho. Metodologia: Estudo descritivo com abordagem quantitativa, realizado junto a enfermeiros atuantes na atenção primária à saúde de um município da região sul do Brasil, sendo instrumento de coleta de dados a versão resumida do Job stress scale. Os resultados desse estudo foram analisados segundo o esquema do modelo de Demanda-Controle de Karasek. Resultados: Participaram do estudo 28 enfermeiros, predominantemente, mulheres $(92,80 \%)$, casadas ou vivendo com companheiro $(67,85 \%)$, com filhos $(64,28 \%)$, na faixa etária 30 a 41 anos $(53,57 \%)$. De maneira geral, os enfermeiros investigados eram profissionais que trabalhavam há muito tempo no mesmo setor, com dupla jornada; demonstraram perceberem-se sujeitos a situações de estresse, com alto controle sobre as mesmas; não se percebiam influenciados pela contribuição de estresse em seu ambiente de trabalho; e demonstraram ser relevante o apoio social por chefes e colegas. Correlações lineares de Person entre as variáveis sociodemográficas e ocupacionais predominantes, demonstraram-se não significativas. Conclusões: Embora a população estudada atue em setor com alta demanda psicológica, que contribui para o desencadeamento de estresse global, são profissionais que tem alto controle sobre a situação do trabalho.
\end{abstract}

Palavras-chave: Enfermagem; Estresse; Atenção primária à saúde.

\begin{abstract}
Introduction: Nurses working in primary health care are professionals potentially susceptible to the development of stress. In their daily lives, they face conflicting situations such as overwork and accumulation of tasks that cause physical and mental strain. Objetive: To analyze the perception of Brazilian nurses working in primary health care about the contribution of stress in the workplace. Methodology: Descriptive study with a quantitative approach, carried out with nurses working in primary health care in a municipality in the southern region of Brazil, with the summary version of the Job stress scale being a data collection instrument. The results of this study were analyzed according to the Karasek Demand-Control model. Results: 28 nurses participated in the study, predominantly women
\end{abstract}


$(92.80 \%)$, married or living with a partner $(67.85 \%)$, with children $(64.28 \%)$, aged 30 to 41 years $(53.57 \%)$. In general, the nurses investigated were professionals who had worked for a long time in the same sector, with a double shift; demonstrated to perceive themselves subject to stressful situations, with high control over them; they were not perceived to be influenced by the stress contribution in their work environment; and demonstrated social support by bosses and colleagues to be relevant. Linear correlations of Person between the predominant sociodemographic and occupational variables, were not significant. Conclusions: Although the studied population works in a sector with high psychological demand, which contributes to the triggering of global stress, they are professionals who have high control over the work situation.

Keywords: Nursing; Stress; Primary health care.

\section{Resumen}

Introducción: Las enfermeras que trabajan en la atención primaria de salud son profesionales potencialmente susceptibles al desarrollo de estrés. En su vida diaria se enfrentan a situaciones conflictivas como el exceso de trabajo y la acumulación de tareas que les provocan tensión física y mental.Objetivo: Analizar la percepción de enfermeras brasileñas que trabajan en la atención primaria de salud sobre la contribución del estrés en el lugar de trabajo. Metodologia: Estudio descriptivo con abordaje cuantitativo, realizado con enfermeras que laboran en la atención primaria de salud en un municipio de la región sur de Brasil, siendo la versión resumida de la escala de estrés laboral un instrumento de recolección de datos. Los resultados de este estudio se analizaron según el modelo Karasek Demand-Control. Resultados: Participaron en el estudio 28 enfermeras, predominantemente mujeres (92,80\%), casadas o conviviendo en pareja (67,85\%), con hijos (64,28\%), de 30 a 41 años $(53,57 \%)$. En general, las enfermeras investigadas eran profesionales que habían trabajado durante mucho tiempo en el mismo sector, con doble jornada; demostraron percibirse sujetos a situaciones estresantes, con alto control sobre ellas; no se percibía que estuvieran influenciados por la contribución del estrés en su entorno laboral; y el apoyo social demostrado por jefes y colegas para ser relevante. Las correlaciones lineales de Person entre las variables sociodemográficas y ocupacionales predominantes resultaron insignificantes. Conclusiones: Si bien la población estudiada trabaja en un sector con alta demanda psicológica, lo que contribuye al desencadenamiento del estrés global, son profesionales que tienen un alto control sobre la situación laboral.

Palabras clave: Enfermeria; Estrés; Atención primaria de salud.

\section{Introdução}

Estresse é uma reação complexa e global do organismo humano, envolvendo componentes físicos, psicológicos, mentais e hormonais, desencadeada por um grupo de reações físicas, tanto no âmbito pessoal como profissional (Hoppe, 2014). No âmbito profissional é proveniente da inserção do trabalhador num contexto adverso, uma vez que, ao mesmo tempo que o trabalho deveria ser fonte de satisfação e realização pessoal, pode trazer insatisfação, desinteresse e frustração. Sobretudo, profissionais de saúde são trabalhadores potencialmente susceptíveis ao desenvolvimento de estresse (Oliveira e Cunha, 2014).

Entre os profissionais de saúde potenciais ao desenvolvimento de estresse destaca-se o enfermeiro (Oliveira e Cunha, 2014; Jordão, 2017), em especial aquele que trabalha no campo da Atenção Primária à Saúde (APS). Na sua linha de trabalho, os enfermeiros estabelecem uma ampla gama de relacionamentos com pacientes e seus familiares, que geram diferentes tipos de tensão, levando a várias situações estressantes, que podem afetar o equilíbrio e a personalidade desses profissionais como um todo, refletindo no nível físico, emocional, psicológico e social (Starc, 2018).

O trabalho na APS se constitui num conjunto de ações de saúde de âmbito individual e coletivo com importante atuação do enfermeiro. Enfermeiros atuantes na APS geralmente desenvolvem ações centradas na organização, gestão de processos de trabalho com um foco maior no atendimento às necessidades das famílias, assistência na atenção aos indivíduos em seu contexto familiar e comunitário (Lorenz e Guirardello, 2014). Fatores como composição insuficiente de equipes, baixos salários e inadequação da estrutura física das unidades têm sido considerados como geradores de insatisfação e estresse entre esses trabalhadores (Merces et al, 2015).

Considerando os danos que o estresse possa desencadear junto ao trabalho do enfermeiro na APS, surgiu o interesse pela realização dessa pesquisa, que partiu da seguinte questão: Como enfermeiros brasileiros atuantes na APS percebem a contribuição do estresse no ambiente de trabalho? Esta pesquisa teve como objetivo analisar a percepção de enfermeiros brasileiros atuantes na Atenção Primária à Saúde sobre a contribuição do estresse no ambiente de trabalho. 


\section{Metodologia}

Trata-se de um estudo descritivo de natureza quantitativa sendo suporte metodológico Fantinato (2015) e Marconi e Lakatos (2012), realizado junto às unidades de APS de um município localizado na região sul do Brasil.

Foram convidados para participar da pesquisa 31 enfermeiros, sendo os convites por e-mails e contatos de whatsapp. Os critérios de inclusão foram: ser enfermeiro com vínculo empregatício na APS por um período mínimo de um ano. Foram excluídos do estudo enfermeiros que, no período da coleta de dados, estavam afastados do trabalho por motivo de férias, licenças médicas ou faltas.

A coleta de dados foi realizada entre os meses de maio e junho de 2018, utilizando como instrumento o Job stress scale versão resumida, o qual envolveu a percepção de enfermeiros atuantes na APS quanto a contribuição de estresse no ambiente de trabalho. Além disso, foi elaborado um instrumento para caracterização dos sujeitos da pesquisa, abrangendo o perfil sociodemográfico e ocupacional dos enfermeiros, segundo sexo, idade, estado civil, presença de filhos, tempo de atuação na APS e atividades desenvolvidas em outro setor.

O Job stress scale é uma versão reduzida do questionário originalmente elaborado por Karasek (49 perguntas). No Brasil Job stress scale versão reduzida foi adaptada para o português por Alvesa et $\mathrm{al}^{(8)}$. A versão reduzida contém 17 questões, cinco para avaliar demanda, seis para avaliar controle e seis para apoio social. Dentre as perguntas que avaliam demanda, quatro referem-se a aspectos quantitativos, como tempo e velocidade para realização do trabalho. Dentre as questões referentes ao controle, quatro se referem ao uso e desenvolvimento de habilidades, como possibilidade de aprender coisas novas no trabalho, habilidade ou conhecimentos especializados. Para as dimensões que avaliam demanda e controle as opções de resposta são apresentadas variando entre "frequentemente", "às vezes", "raramente" e "nunca/quase nunca". O bloco referente ao apoio social contém questões sobre as relações com colegas e chefes. Para todos os itens, uma escala tipo Likert, com variação de intensidade correspondente de 1 a 4, mediu a contribuição de estresse no ambiente de trabalho que a situação provoca.

Os resultados referentes à percepção de enfermeiros atuantes na APS quanto à contribuição de estresse no ambiente de trabalho foram distribuídos estatisticamente, sendo a análise realizada segundo o esquema do modelo de Demanda-Controle de Karasek (Alvesa et al, 2004). Nesse modelo, escores médios foram alocados em quatro quadrantes de forma a expressar as relações entre controle e demanda psicológica, e classificados em baixo desgaste, ativo, passivo e alto desgaste. A coexistência de grandes demandas psicológicas com baixo controle sobre o processo de trabalho gera alto desgaste ("job strain”) no trabalhador, com efeitos nocivos à sua saúde. Também nociva é a situação que conjuga baixas demandas e baixo controle (trabalho passivo), na medida em que podem gerar perda de habilidades e desinteresse. Por outro lado, quando altas demandas e alto controle coexistem, os indivíduos experimentam o processo de trabalho de forma ativa, ou seja, ainda que as demandas sejam excessivas, elas são menos danosas, na medida em que o trabalhador pode escolher como planejar suas horas de trabalho de acordo com seu ritmo biológico e criar estratégias para lidar com suas dificuldades. A situação "ideal”, de baixo desgaste, conjuga baixas demandas e alto controle do processo de trabalho.

Foi utilizada a correlação linear de Person (Marconi e Lakatos (2012) entre os resultados dos dados sociodemográficos e ocupacionais predominantes.

A pesquisa foi aprovada pelo Comitê de Ética em Pesquisa de uma universidade pública, sob o parecer $\mathrm{n}^{\circ}$ 2.712.182/2018.

\section{Resultados}

Dos 31 convidados para participar do estudo, uma estava de licença maternidade e dois de férias no momento do período de coleta de dados. Assim, participaram 28 enfermeiros. Houve predomínio de mulheres (92,80\%), casadas ou vivendo 
com companheiro (67,85\%), com filhos (64,28\%), na faixa etária 30 a 41 anos (53,57\%), atuantes na APS por um período superior há 10 anos $(67,85 \%)$, e exercendo atividades em outro setor além do trabalho na APS, caracterizando dupla jornada de trabalho $(64,28 \%)$.

A percepção de enfermeiros atuantes na APS quanto à contribuição de estresse no ambiente de trabalho e o apoio recebido envolvendo colegas e chefes, estão apresentadas nas Figuras 1 e 2, sendo as contribuições de estresse classificados em baixo desgaste, ativo, passivo e alto desgaste demonstrados na Figura 1; e o ambiente de trabalho envolvendo colegas e chefes, discriminado como calmo e agradável, com boa relação entre chefes e colegas, apoio dos colegas, compreensão dos colegas sobre um dia ruim, boa relação com os chefes e gostar de trabalhar com os colegas, na Figura 2.

Figura 1. Distribuição da percepção de enfermeiros atuantes em APS quanto a contribuição de estresse no ambiente de trabalho.

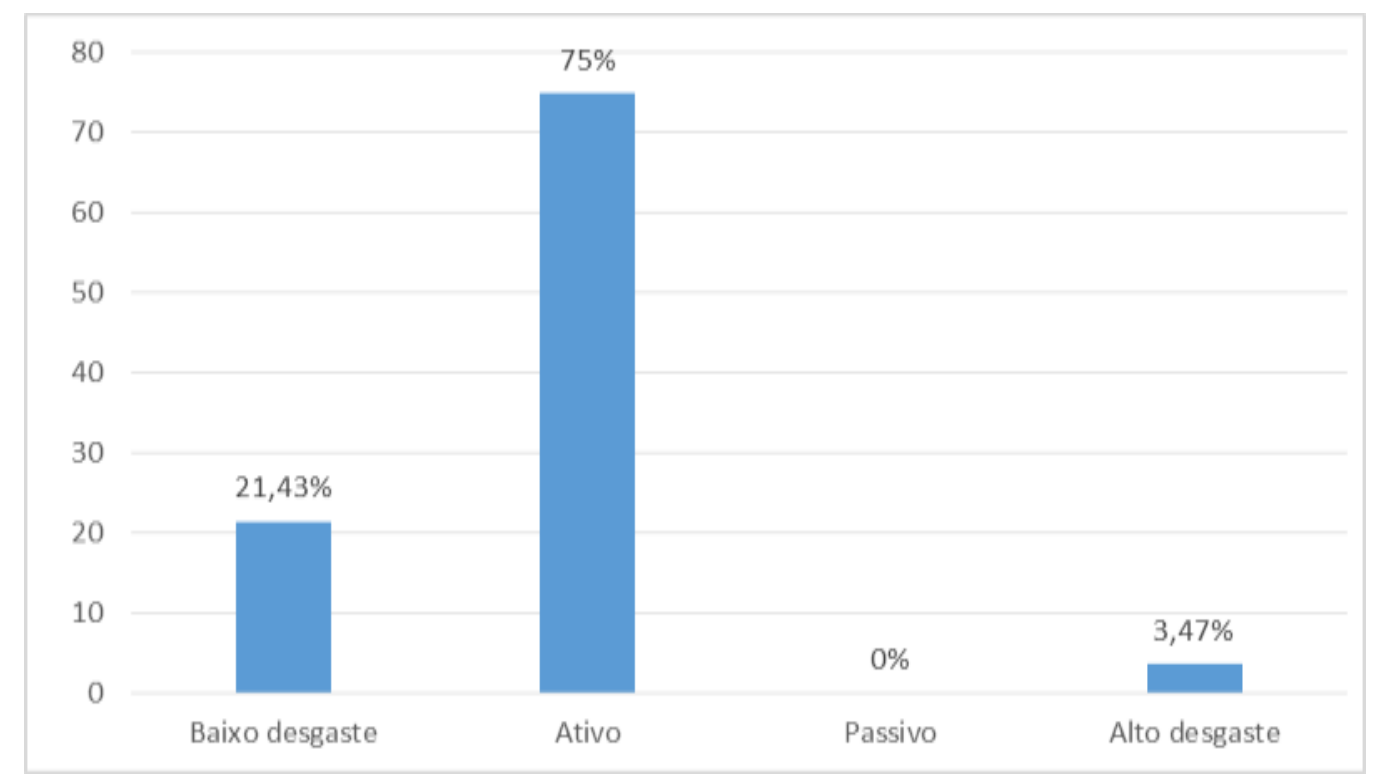

Fonte: Autores.

Figura 2. Distribuição da percepção de enfermeiros atuantes na APS quanto ao apoio recebido no ambiente de trabalho envolvendo colegas e chefes.

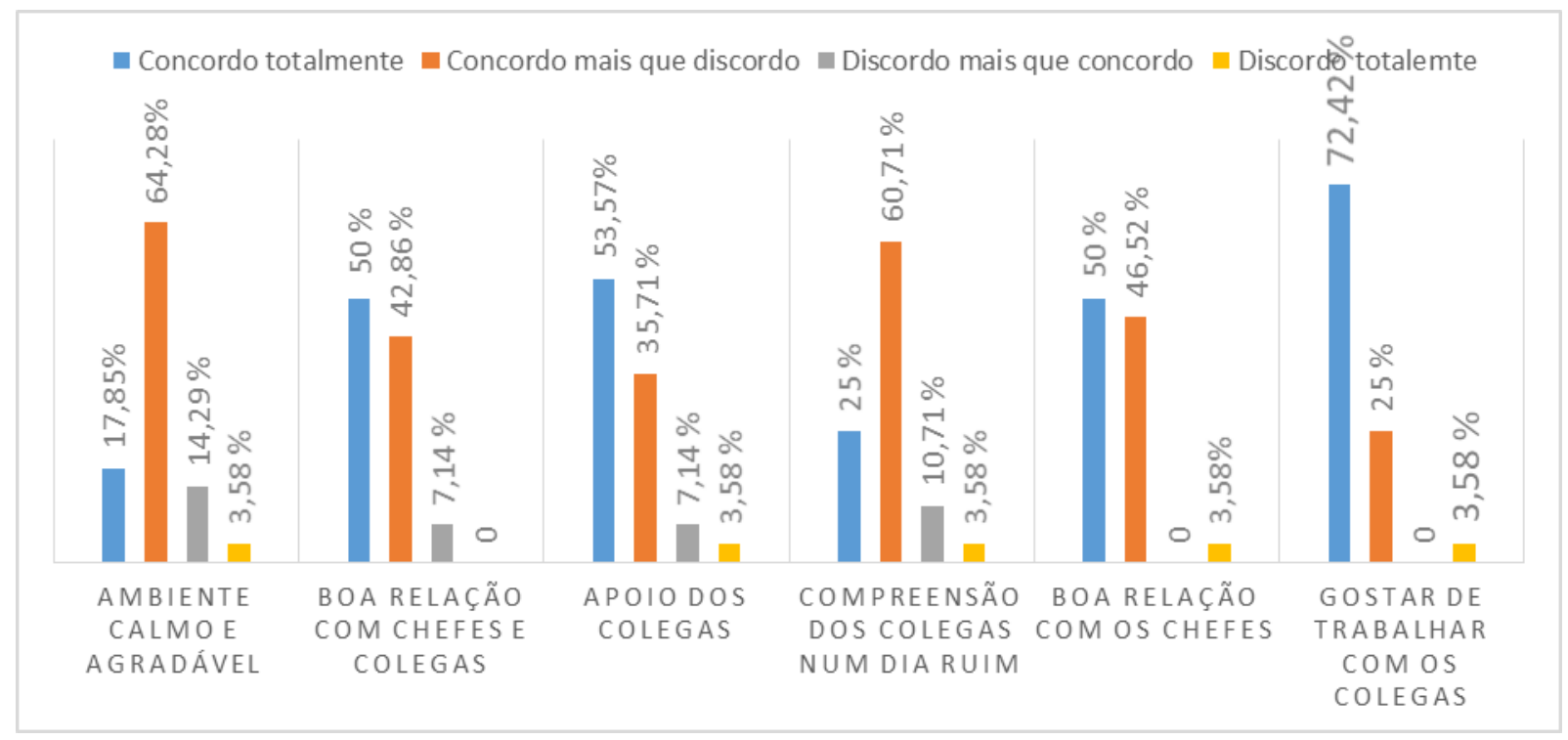

Fonte: Autores. 
Verificou-se a situação de baixo desgaste, que reflete situações de baixas demandas psicológicas e alto controle do processo de trabalho, considerado um resultado benéfico. Embora em menor percentual, a situação de alto desgaste também foi evidenciada, demonstrando a coexistência de grandes demandas psicológicas com baixo controle sobre o processo de trabalho geral nesses profissionais, o que pode acarretar em efeitos nocivos à saúde.

Os enfermeiros demonstraram perceber que existe um ambiente calmo e agradável no trabalho; relacionar-se bem uns com os outros; contar com o apoio de seus colegas; ser compreendido por seus colegas caso estejam num dia ruim; ter uma boa relação com os chefes no trabalho; e, gostar de trabalhar com seus colegas.

Correlações lineares de Person entre os resultados das variáveis dos dados sobre a percepção de enfermeiros quanto à contribuição de estresse no ambiente de trabalho e a atividade desenvolvida em outro setor além do trabalho e o tempo de atuação na APS, estão apresentadas nas Tabelas 1,2,3 e 4.

Tabela 1. Matriz de Correlação entre a percepção de enfermeiros quanto a contribuição de estresse no ambiente de trabalho e o tempo de atuação na APS.

\begin{tabular}{|l|c|c|}
\hline & Percepção de Estresse & Tempo em anos na APS \\
\hline Percepção de Estresse & 1,000 & \\
\hline Tempo em anos na APS & $-0,230$ & 1,000 \\
\hline
\end{tabular}

Fonte: Autores.

Tabela 2. Matriz de P entre a percepção de enfermeiros quanto a contribuição de estresse no ambiente de trabalho e o tempo de atuação na APS.

\begin{tabular}{|l|c|c|}
\hline & Percepção de Estresse & Tempo em anos na APS \\
\hline Percepção de Estresse & 1,000 & \\
\hline Tempo em anos na APS & 0,239 & 1,000 \\
\hline
\end{tabular}

Fonte: Autores.

Tabela 3. Matriz de Correlação entre a percepção de enfermeiros quanto a contribuição de estresse no ambiente de trabalho e atividade desenvolvida em outro setor além do trabalho na APS.

\begin{tabular}{|l|c|c|}
\hline & Percepção de Estresse & Trabalho além à APS \\
\hline Percepção de Estresse & 1,000 & \\
\hline Trabalho além à APS & 0,073 & 1,000 \\
\hline
\end{tabular}

Fonte: Autores.

Tabela 4. Matriz de P entre a percepção de enfermeiros quanto a contribuição de estresse no ambiente de trabalho e atividade desenvolvida em outro setor além do trabalho na APS.

\begin{tabular}{|l|c|c|}
\hline & Percepção de Estresse & Trabalho além à APS \\
\hline Percepção de Estresse & 1,000 & 0,711 \\
\hline Trabalho além à APS & 0,711 & 1,000 \\
\hline
\end{tabular}

Fonte: Autores.

Observou-se nas Tabelas 1 e 2 que não houve correlação entre a percepção de enfermeiros quanto à contribuição de estresse no ambiente de trabalho e o tempo de atuação na APS. Assim como, nas Tabelas 3 e 4, evidenciou-se que não houve 
correlação entre a percepção de enfermeiros quanto à contribuição de estresse no ambiente de trabalho e a atividade desenvolvida em outro setor além do trabalho na APS.

\section{Discussão}

O trabalho na APS é um setor da saúde que exige muitas atribuições às suas equipes, com elevado grau de exigências e responsabilidades (Leonelli et al, 2017; Martins et al, 2014). O planejamento e as ações dos serviços estão em permanente construção, e podem promover situações de estresse entre aqueles que nessa área trabalham (Lorenz \& Guirardello, 2014; Merces et al, 2016).

No modelo de APS brasileiro os profissionais de saúde possuem um papel central, pois ao utilizarem tecnologias leves para resolução de problemas complexos necessitam estar em contato direto com pacientes e comunidades. $\mathrm{O}$ relacionamento interpessoal no trabalho em saúde é apontado por Martins et al (2014) como a principal classe de estressores psicossociais. Relaciona-se a isto o fato de os profissionais de saúde lidarem diretamente com demandas complexas dos usuários que atendem. Não obstante, a exposição crônica aos estressores dessa natureza pode desencadear intensa exaustão emocional, redução da satisfação no trabalho e dificuldades para atender de forma humanizada aos demais usuários do sistema de saúde.

Entre os profissionais de saúde susceptíveis aos fatores de estresse destacam-se os trabalhadores de enfermagem, em especial as mulheres. A Enfermagem tem se caracterizado como uma profissão feminina tendo em vista o número expressivo de mulheres que integram a profissão. Esta assertiva pode ser constatada no Brasil a partir de um estudo realizado pelo Conselho Federal de Enfermagem (COFEN), em parceria com a Fundação Oswaldo Cruz (FIOCRUZ) em 2016, no qual evidenciou-se que a Enfermagem alcança um contingente de sua força de trabalho composto de $85,1 \%$ de mulheres (Machado, 2017).

Destaca-se no presente estudo a predominância de mulheres enfermeiras exercendo atividades em outro setor além do trabalho na APS, caracterizando dupla jornada de trabalho. A área da enfermagem caracterizada como uma profissão feminina, não é de todo benéfica, pois uma enfermeira, trabalhadora em especial na APS, sofre dupla jornada como a maioria das mulheres. Isto é, além de seu trabalho remunerado fora de casa, existe o trabalho doméstico, composto pelo cuidado com os filhos e com a casa, o que pode contribuir para prejuízos na produção adequada da assistência de enfermagem (Cيrdeiro \& Araújo, 2017).

Compreende-se que independe do gênero, o enfermeiro da APS desenvolve seu trabalho tanto na unidade de saúde, junto à equipe de profissionais, quanto na comunidade, assistindo os indivíduos e famílias que necessitam de cuidado. As determinadas situações na relação trabalhador-usuário demandam gasto de energia e adaptação, como o contato direto com a realidade e/ou o sofrimento do próximo, elementos próprios do tipo de trabalho, como uma certa identificação e os laços afetivos que, frequentemente se estabelecem. Acredita-se que se essas situações, somadas às características individuais de cada profissional, não forem trabalhadas internamente pelo profissional, podem desencadear o processo de estresse.

O enfermeiro, na sua função, deve buscar o equilíbrio da equipe, e isso, muitas vezes, pode se tornar desgastante, tornando-se mais um dos fatores desencadeantes de sintomas de estresse. Além disso, consideram-se fatores estressores a falta de estrutura física para o desempenho de atividades; a ausência de reconhecimento profissional; a alta demanda de atendimentos; a carga horária elevada; a insatisfação na remuneração; o trabalho repetitivo; e a falta de segurança no trabalho. Esses fatores estressores podem incapacitar os enfermeiros na realização de seus trabalhos, interferindo na relação do profissional com a população, levando a um prejuízo na assistência prestada, assim como afetar negativamente a qualidade de vida desses trabalhadores (Siqueira et al, 2013). Sob uma visão geral, a preocupação prepondera, quando se pensa em dupla jornada no trabalho. 
Enfermeiros, no intuito de superar as adversidades do seu trabalho, buscam motivação, como o dinheiro e o conhecimento, para seguir uma jornada dupla de trabalho, desafiando os fatores extrínsecos e intrínsecos que surgem constantemente (Dalri, 2014). A dupla jornada de trabalho pode se tornar elemento que propicia desgaste e sofrimento ao enfermeiro, constituindo uma dimensão importante na qualidade laboral, repercutindo na segurança e saúde do trabalhador e nos aspectos pessoais e familiares, isto porque ao terem que sair de uma instituição para a outra, muitas vezes sem a pausa necessária, sofrem situações de amplo desgaste físico, com prejuízo social pelo tempo escasso para o convívio familiar, o que pode interferir em alguns aspectos referentes à qualidade de vida dessa população específica (Oliveira \& Cunha, 2014).

Ressalta-se que na APS não é cabível uma jornada de trabalho para além das oito horas diárias e finais de semana, o que pode possibilitar a existência de múltiplos vínculos para os profissionais enfermeiros lotados nessa área (Cordeiro \& Araújo, 2017). Contudo, entre os trabalhadores desse estudo, obteve-se resultados positivos no que diz respeito a relação entre a equipe e os profissionais gestores no processo de trabalho. Assim, considerou-se relevante o apoio social recebido pelos enfermeiros no ambiente de trabalho envolvendo colegas e chefes.

O suporte ou apoio social laboral é o apoio ofertado por indivíduos mais próximos dentro do ambiente laboral, como uma companhia, um afeto, ou simplesmente uma palavra de encorajamento, para que o sujeito se sinta protegido em várias situações, principalmente, as estressogênicas. O nível de suporte ou apoio social é um dos fatores protetores contra os efeitos deletérios do estresse laboral, além de operar como intermediário entre as demandas psicológicas e o controle no ambiente psicossocial do trabalho, pois uma rede de apoio faz com que as demandas psicológicas sejam repartidas (Velasco, 2014). Em contrapartida, para Velasco (2014), o baixo apoio social pode inclusive, influenciar nos relacionamentos interpessoais do trabalho e em outros âmbitos, já que é um potencializador do estresse laboral e repercute em todas as áreas de vida do trabalhador podendo acarretar uma maior chance de adoecimento.

Compreende-se que no presente estudo, o predomínio de informações demonstrando adequado apoio social no ambiente de trabalho envolvendo colegas e chefes, seja o fator contribuidor no autocontrole das situações de estresse percebidas pelos enfermeiros. Destaca-se a evidência constatada mediante a análise das relações entre controle e demanda psicológica classificadas segundo o modelo de Demanda-Controle de Karasek (Alvesa,et al, 2004), que permitiu verificar entre os investigados a presença de altas demandas psicológicas e alto controle, assim como, não haver correlações lineares de Person significativas entre os resultados das variáveis dos dados sociodemográficos e ocupacionais predominantes

\section{Conclusão}

Os enfermeiros estudados, atuantes em setor com alta demanda psicológica, contribuidora para o desencadeamento de estresse global, demonstraram ter bom relacionamento com chefes e colegas, e alto controle sobre a situação do trabalho. $\mathrm{O}$ fato de trabalharem muito tempo no mesmo setor e ou serem sujeitos de dupla jornada, não os tornou influenciados pela contribuição de estresse no ambiente de trabalho.

Espera-se que os resultados desse estudo possam desencadear a expansão de conhecimentos junto a temática estresse e o trabalho de enfermeiros atuantes na APS, subsidiando na perspectiva laboral a construção de práticas seguras envolvendo a saúde mental no contexto do trabalho de enfermagem; assim como, que sirva de suporte teórico na abordagem deste tema a produção de estudos científicos futuros.

\section{Referências}

Alvesa, M. G. M., Chorb, D., Faersteinc, E., Lopesc, C. S., \& Werneckd, G. L. (2004). Short version of the "job stress scale": a Portuguese-language adaptation. Revista de Saúde Pública, 38(2):1-7. https://scielosp.org/pdf/rsp/2004.v38n2/164-171/en

Cordeiro, T. M. S. C., \& Araújo, T. M. (2017) Prevalência da capacidade para o trabalho inadequada entre trabalhadores de enfermagem da atenção básica à saúde. Rev Bras Med Trab., 15(2):150-7. https://docs.bvsalud.org/biblioref/2017/08/848122/rbmt-v15n2_150-157.pdf 
Dalri, R. C. M. B., Silva, L. A., Mendes, A. M. O. C., \& Robazzi, M. L. C. C. (2014). Nurses' workload and its relation with physiological stress reactions. Rev. Latino-Am. Enfermagem, 22(6), 959-965. https://www.scielo.br/pdf/rlae/v22n6/0104-1169-rlae-22-06-00959.pdf

Fantinato, M. (2015). Métodos de pesquisa. SEAD.

Hoppe, T. N. (2014). Estresse ocupacional: Percepções de colaboradores de uma instituição de ensino superior. Biblioteca digital da UNIVATES BDU. Obtido em: https://www.univates.br/bdu/handle/10737/407

Jordão, N. A. F. (2017). Condições de trabalho e absenteísmo por doença entre os profissionais de enfermagem da rede municipal de saúde de Belo Horizonte. Dissertação de Mestrado. Repositório: Universidade Federal de Minais Gerais. https://repositorio.ufmg.br/bitstream/1843/ANDOAM9KJR/1/disserta_o.15.04.2017.final.pdf

Leonelli, L. B., Andreoni, S., Martins, P., Kozasa, E. H., Salvo, V. L., Sopezki, D. et al. (2017). Perceived stress among Primary Health Care Professionals in Brazil. Rev Bras Epidemiol. 20(2): 286-98. https://www.scielo.br/pdf/rbepid/v20n2/en_1980-5497-rbepid-20-02-00286.pdf

Lorenz, V. R. \& Guirardello, E. B. (2014). The environment of professional practice and Burnout in nurses in primary healthcare. Revista Latino-Americana de Enfermagem, 22(6):926-33. https://www.scielo.br/pdf/rlae/v22n6/0104-1169-rlae-0011-2497.pdf

Machado, M. H. (2017). Perfil da Enfermagem no Brasil: relatório final. NERHUS - DAPS - ENSP/Fiocruz, 1:748 p. http://www.cofen.gov.br/perfilenferm agem/pdfs/relatoriofinal.pdf

Marconi, M. A., \& Lakatos, E. M. (2012). Técnicas de pesquisa. Atlas. 2012.

Martins, L. F., Laport, T. J., Menezes, V. P., Medeiros, P. B., \& Ronzani, T. M. (2014). Esgotamento entre profissionais da Atenção Primária à Saúde. Ciência \& Saúde Coletiva. 19(12):4939-4750. https://www.scielo.br/pdf/csc/v19n12/1413-8123-csc-19-12-04739.pdf

Merces, M. C., Cordeiro, T. M. S. C., Santana, A. I. C., Lua, I., Silva, D. S., Alves, M. S., Luz, M. S., \& Oliveira Júnior, A. (2016). Síndrome de Burnout em trabalhadores de enfermagem da Atenção Básica á Saúde. Revista Baiana de Enfermagem, 30(3): 1-9. https://periodicos.ufba.br/index.php/enfermage m/article/view/15645/pdf_64

Ministério da Saúde. Portaria $\mathrm{n}^{\circ}$ 2.436. Aprova a Política Nacional de Atenção Básica. Brasília. 2017. https://bvsms.saude.gov.br/bvs/saudelegis/gm /2017/prt2436_22_09_2017.html

Oliveira, R. J., \& Cunha, T. (2014). Estresse do profissional no ambiente de trabalho: causas e consequências. Caderno saúde e desenvolvimento, 3 (2):78-93. Obtido em: file:///C:/Users/Usuario/Downloads/302-1224-1-PB.pdf

Siqueira, G. F. F., Barrêto, A. J. R., Menezes, M. S., Alves, S. R. P., \& Freitas, F. F. Q. (2013). Trabalho do enfermeiro na Atenção Primária em Saúde: conhecimento dos fatores estressores. Rev. Ciênc.Saúde Nova Esperança, 11(2), 72-85. https://revista.facene.com.br/index.php/revistane/article/view/507/400

Starc, J. (2018). Stress factors among Nurses at the primary and secondary level of public sector health care: The case of Slovenia. Open Access Macedonian Journal of Medical Sciences, 6(2):416-22: https://www.ncbi.nlm.nih.gov/pmc/articles/PMC5839460/pdf/OAMJMS-6-416.pdf

Velasco, A. R. (2014). O estresse laboral dos trabalhadores de enfermagem das equipes de saúde da família. Dissertação de Mestrado. Repositório: Universidade Federal do Estado do Rio de Janeiro. http://www.unirio.br/ppgenf/dissertacoes/dissertacoes-ppgenf-unirio-ano-2014/dissertacao-aline-ramos 